J3eA, Journal sur l'enseignement des sciences et technologies de l'information et des systèmes, Volume 4, Hors-Série 4, 5 (2005)

DOI : http://dx.doi.org/10.1051/bib-j3ea:2005855

(C) EDP Sciences, 2005

\title{
La Résonance Magnétique Nucléaire : sujet de développements et outil de diagnostic
}

\author{
A. Briguet \\ Laboratoire de Résonance Magnétique Nucléaire, Méthodologie et Instrumentation \\ en Biophysique, UMR 5012, CNRS \\ Université Claude Bernard Lyon I \\ ESCPE Lyon
}




\title{
COMMUNICATION DANS LES JOURNEES DU CLUB EEA
}

\author{
« Diagnostic en Electronique, Electrotechnique et Automatique »
}

\author{
17-18 Mars 2005 Lyon
}

\section{La Résonance Magnétique Nucléaire : sujet de développements et outil de diagnostic}

André Briguet, Laboratoire de Résonance Magnétique Nucléaire, Méthodologie et Instrumentation en Biophysique, UMR 5012, CNRS, Universitél Claude Bernard Lyon1, ESCPE Lyon.

\section{Introduction}

La résonance magnétique nucléaire $(\mathrm{RMN})$ est, à l'origine, une technique de détection et de mesure du paramagnétisme nucléaire utilisée depuis bientôt soixante années. Mais son champ d'application principal ne sert pas, ou alors très peu, à traiter du magnétisme lui même : ce champ d'application est celui, traditionnel, de la Chimie et celui de l'Imagerie Médicale qui a pris une place considérable au cours des vingt dernières années. Dans cette présentation qui débute par un très bref rappel du principe de la RMN, on expliquera tout aussi rapidement ce que sont la spectroscopie et l'imagerie fondées sur l'emploi de la RMN tout en insistant sur quelques aspects techniques et instrumentaux. On essaiera également, à partir d'exemples tirés du laboratoire, de situer la RMN parmi les techniques possibles de contrôle non destructif dans d'autres domaines que le biomédical.

\section{Principe général}

On sait que certains noyaux possèdent un moment magnétique lié à une propriété microscopique particulière, l'existence d'un moment de spin. Un ensemble de noyaux ainsi doués de magnétisme et formant un échantillon macroscopique ne présente pas d'aimantation sauf s'il est plongé dans un champ magnétique que l'on choisit statique, et que l'on appelle aussi champ directeur. En effet, à la température ordinaire, de l'ordre de 300K , l'aimantation ainsi créée est dans le même sens que le champ appliqué, elle lui est proportionnelle et elle est inversement proportionnelle à la température. On est bien dans le cas du paramagnétisme. Toutefois cette aimantation est tellement faible qu'elle est masquée par l'aimantation du milieu qui forme l'échantillon, aimantation qui a pour origine les orbitales électroniques pleines donnant lieu au diamagnétisme et qui est le cas le plus généralement rencontré. C'est alors qu'il est possible de détecter sélectivement l'aimantation nucléaire à l'aide d'une expérience de résonance qui est un procédé assez général utilisé pour mettre en évidence des propriétés de faible ampleur. Cette sélectivité va se retrouver au niveau de chaque noyau, qui, s'il présente un moment magnétique de spin, possédera une fréquence de résonance propre dans un champ donné. C'est ainsi que le proton résonne à $400 \mathrm{MHz}$ dans un champ de 9,4 teslas contre $100 \mathrm{MHz}$ environ pour les noyaux de carbone 13 dans ce même champ magnétique. Cette distinction entre les noyaux qui sont capables de donner lieu au phénomène de RMN est alors traduite par la fréquence de Larmor $f$ qui caractérise le mouvement de précession d'une aimantation nucléaire autour de la direction du champ 
magnétique, une fois qu'elle est écartée de sa position d'équilibre thermique qui se trouve dans le sens de ce champ, noté $B$.

$$
2 \pi f=\gamma B
$$

où $\gamma$ est appelé rapport gyromagnétique des noyaux considérés.

En effectuant ce mouvement de précession et tout en reprenant progressivement sa position d'équilibre (c'est le phénomène de relaxation), l'aimantation est donc en mesure d'induire un signal électrique dans une bobine. Ce signal sera perçu au mieux si on lui affecte une bande spectrale étroite permettant de rejeter un maximum d'énergie de bruit, c'est à dire si l'on accorde la fréquence de résonance électrique du capteur à celle du mouvement de précession. C'est l'interprétation instrumentale du terme de résonance. Compte tenu de l'ordre de grandeur des rapports gyromagnétiques de noyaux qui se comptent en dizaine de millions de tours par seconde et par tesla, on constate que les fréquences de travail sont dans le domaine des radiofréquences, typiquement jusqu'à $1 \mathrm{GHz}$ actuellement puisque les champs les plus élevés et stables que l'on sait créer sont de l'ordre de 23 teslas. D'ailleurs, et par réciprocité, on pourra justement déplacer l'aimantation de sa position d'équilibre thermique en utilisant une excitation radiofréquence à la fréquence de Larmor, c'est à dire en appliquant un champ magnétique variable dans le temps à cette fréquence, et dirigé perpendiculairement au champ statique. Ceci signifie que le même résonateur électrique peut servir d'excitateur puis de récepteur. Dans un premier temps le résonateur, qui peut entourer l'échantillon, est alors le siège d'une impulsion radiofréquence assez intense permettant d'engendrer le mouvement de précession de l'aimantation. Dans un second temps le récepteur recueille une force électromotrice radiofréquence donnant un signal dont le caractère transitoire provient de la relaxation. Schématiquement la détection s'effectue par hétérodynage avec soustraction de fréquence comme en technique radio, ce qui permet de disposer d'un signal amorti, basse fréquence, formé de deux composantes en quadrature. L'échantillonnage de ce signal conduit à des données numériques complexes qui sont analysées par transformation de Fourier discrète. Le fait de pouvoir disposer de ces données complexes présente des avantages importants, comme celui de pouvoir attribuer à la composante de fréquence $+\mathrm{f}$, une information différente de celle qui se trouve à la fréquence - $\mathrm{f}$. Par conséquent il est possible de limiter la bande passante utile et de réduire ainsi la puissance du bruit reçue. Par conséquent, le filtre analogique antirepliement situé à l'amont de l'échantillonneur joue un rôle capital car c'est lui qui détermine cette puissance de bruit. Autre élément à prendre en compte est le facteur de bruit du préamplificateur qui conditionne les performances de l'ensemble de la chaîne d'acquisition. 


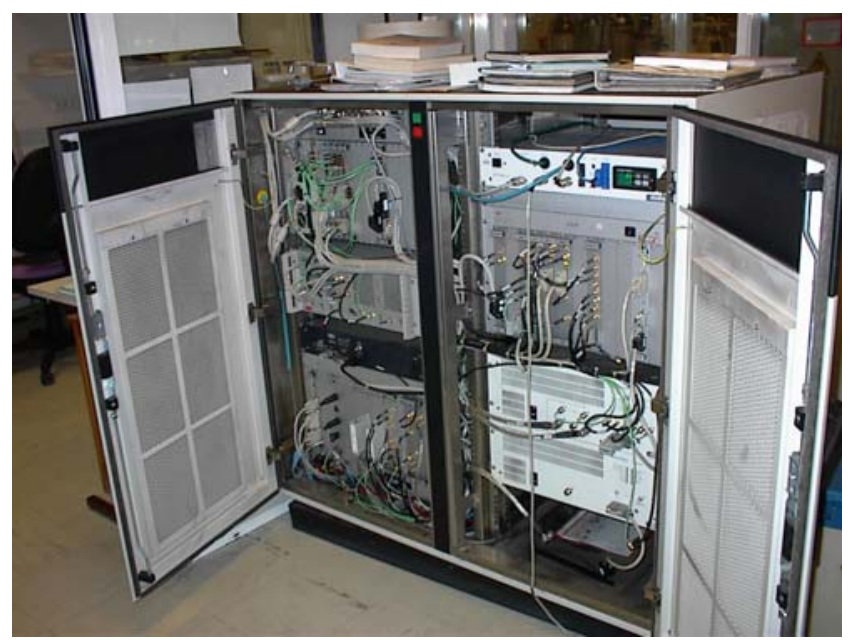

Figure1: Chaîne de mesure d'un système de spectrométrie Bruker (Wissembourg)

\section{Emploi de la RMN en spectroscopie}

C'est avec les applications en spectroscopie que les problèmes instrumentaux concernant la RMN se sont posés à une échelle industrielle. A partir de la fin des années 1950 le besoin d'analyse RMN pour la recherche et pour l'industrie s'est fait ressentir, ce qui a entraîné la nécessité de développer des appareils nombreux et standardisés; ceci a donc engendré une industrie, certes limitée mais très spécialisée, autour de la réalisation d'appareillages de plus en plus fiables et performants. En effet la fréquence de résonance des noyaux est très légèrement modifiée par leur environnement électronique, ce qui traduit en quelque sorte l'effet du groupement fonctionnel auquel ils appartiennent. C'est ainsi que dans l'éthanol les protons de groupement méthyles $(-\mathrm{CH} 3)$ donneront des signaux distincts des signaux correspondant aux protons des groupements méthyléniques (-CH2-). Ce phénomène, appelé « déplacement chimique » est général, il est relativement plus important chez les noyaux notablement entourés d'électrons (carbone 13 par exemple) que dans le cas de l'hydrogène pauvre en électrons. Pour un noyau donné occupant une fonction chimique donnée, la valeur du déplacement chimique est comptée relativement par rapport à la fréquence de résonance moyenne et cette valeur relative est constante quel que soit le champ utilisé. Ceci signifie que l'écartement spectral absolu croît proportionnellement à la valeur du champ statiqu. Mieux encore, et si l'uniformité du champ le permet, on observe en général une structure hyperfine des résonances de ces groupements fonctionnels, structure qui s'interprète aisément à partir de considérations quantiques. Cette démultiplication des raies de résonance trouve son origine dans le fait que les électrons qui participent aux liaisons chimiques ont une probabilité de présence non nulle au contact des différents noyaux concernés par la liaison. C'est ce que l'on appelle le couplage scalaire, dont la mesure, indépendante de la valeur du champ statique, renseigne sur la stéréochimie de la molécule. Enfin les phénomènes de relaxation dans certaines grosses molécules permettent de comparer des distances internucléaires de groupements fonctionnels éloignés sur la chaîne carbonée et par exemple de renseigner sur les repliements de protéines. En effet et à condition d'être suffisamment solubles dans l'eau et de n'avoir pas une trop grand masse moléculaire (inférieure à 25000 Daltons), les protéines peuvent voir leur structure élucidée par des techniques de spectroscopie multidimensionnelle fondées sur la RMN. Comme 
pour toute méthode spectroscopique, l'intérêt réside dans le pouvoir de résolution de la technique, pouvoir qui peut atteindre des valeurs très élevées : par exemple il n'est pas difficile, en milieu liquide, d'obtenir des largeurs de raie de $0,5 \mathrm{~Hz}$ à une fréquence de $500 \mathrm{MHz}$ ce qui donne un pouvoir de résolution de $10{ }^{9}$. Par conséquent on comprend que dans ces conditions il est indispensable de maintenir un très haut degré de stabilité du rapport qui peut exister entre le champ magnétique statique donné par l'aimant et la fréquence du signal radio qui sert à exciter puis à détecter les aimantations nucléaires. Le verrouillage entre les deux grandeurs est alors réalisé électroniquement. L'autre élément technique important réside dans le traitement des données acquises. Bien qu'un bon traitement ne soit pas en mesure de rattraper les erreurs d'une expérience mal conduite, la faiblesse du rapport signal sur bruit impose de recourir à des perfectionnements de méthodes d'analyse qui sont essentiellement fondées sur la transformation de Fourier. On met à profit le fait que la sensibilité peut être améliorée par des techniques de «moyennage » associées, au plan instrumental, à l'emploi de sondes cryogéniques dont le fonctionnement à basse température a pour but de réduire le bruit thermique en provenance du capteur que constitue le résonateur radiofréquence.

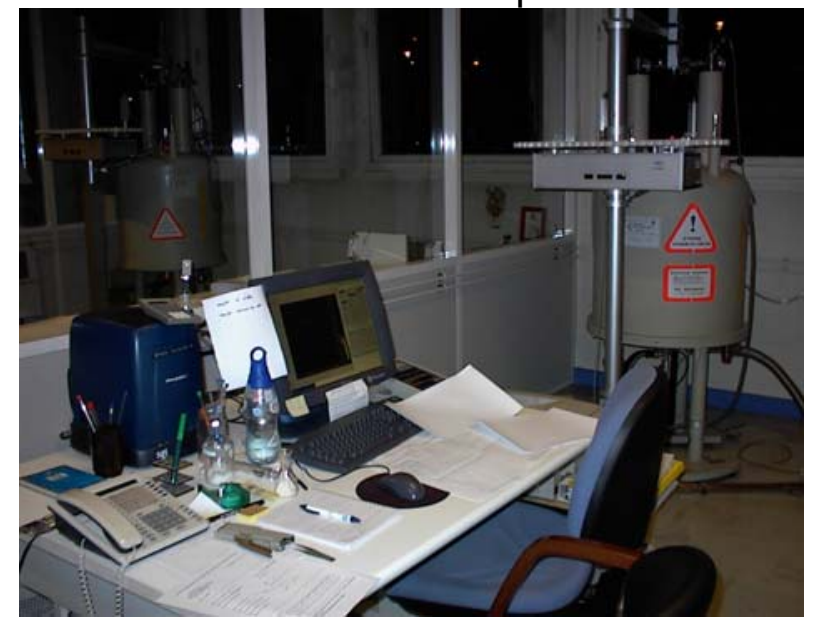

Figure 2: Spectromètre Bruker opérant à $300 \mathrm{MHz}$ pour l'analyse d'échantillons liquides. Au premier plan la console de commande, au fond l'aimant de 7 teslas à champ vertical.

\section{Emploi de la RMN en imagerie}

L'imagerie par RMN a été intuitivement perçue comme réalisable vers la fin des années 1950 par Gabillard qui s'est illustré dans le domaine de l'électronique de puissance, de l'automatique et des transports. A cette époque les moyens de calculs n'étaient adaptés, ni par leur puissance ni par la façon dont on était capable de les gérer. II faut donc attendre 1973 pour que le principe général de l'imagerie par RMN soit démontré et il faut attendre encore un peu pour que s'impose à nouveau l'approche par transformation de Fourier. L'imagerie par RMN est en fait une expérience de très grande simplicité puisque les signaux, qui sont collectés pendant une expérience où le temps qui se déroule est le paramètre important, appartiennent ainsi au domaine réciproque du spectre que l'on va ensuite calculer. II suffit de coder la phase du signal en fonction des positions spatiales pour obtenir une image par des transformations de Fourier du signal enregistré. Cette image sera une image 1D si le codage est fait selon une seule direction à l'aide d'un gradient du champ 
magnétique, ce sera une image 2D si on emploie de manière appropriée deux gradients de directions orthogonales, on aura une image 3D avec trois gradients. On peut même coder par la phase des informations complémentaires: la vitesse d'écoulement ou bien l'information spectroscopique, ce qui permet de disposer d'images 4D dans lesquelles une coupe sera une image 3D dont à son tour une coupe sera une image 2D et dont une coupe sera une image 1D, c'est à dire un profil de l'échantillon selon une direction. Depuis une trentaine d'années un très grand nombre d'expériences différentes par imagerie par RMN ont été élaborées sans jamais calmer l'imagination des chercheurs et des ingénieurs qui proposent constamment de nouvelles approches. Mentionnons l'imagerie ultra rapide qui donne un rendu quasi continu donc une remarquable perception du mouvement, qui a été inventée en 1974 et qui ne fonctionne de manière satisfaisante que depuis quelques années. C'est grâce à un effort considérable de l'industrie et de la recherche en matière de construction d'aimants, de mise au point de systèmes radiofréquence, de moyens d'acquisition du signal pour ne citer que ces quelques cas car la liste est longue, que l'on a abouti à ces résultats. Enfin, il est important de mentionner un élément qui a pu justifier de tels efforts de développement technique; c'est sans doute la très grande plage de contraste possible permise par les techniques d'imagerie RMN. Et, à son tour, cette propriété remarquable a engendré une industrie nouvelle et florissante portant sur l'élaboration des agents de contraste ainsi qu' une recherche dans ce domaine, recherche qui conduit à des exemples de plus en plus innovants et de plus en plus fréquents de visualisation in vivo de l'expression génique, c'est ce que l'on appelle l'imagerie moléculaire.
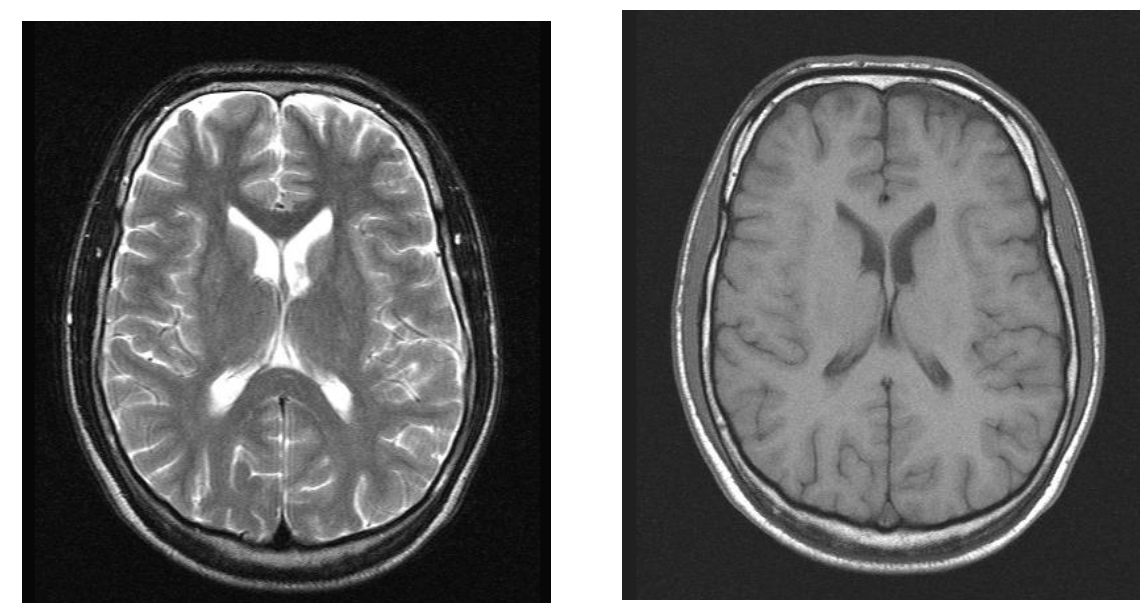

Figure 3: Deux images RMN du même cerveau, observées avec des contrastes opposés en jouant sur les séquences d'observation (cliché Olivier Beuf, UMR 5012)

\section{Quelques enjeux}

Pour simplifier, et en se plaçant du point de vue des spécialistes de l'EEA, on peut mentionner au moins deux cas correspondant à des enjeux sur le plan industriel et sur celui de la recherche : 
L'enjeu technologique et instrumental: il touche principalement des applications, presque banales, de l'électromagnétisme : réaliser des aimants délivrant des champs élevés (supérieurs à 2 teslas, avec de grandes uniformités spatiales sur de vastes volumes : typiquement une variation relative de la valeur du champ inférieure à une partie par million dans une sphère de 30 à $40 \mathrm{~cm}$ de diamètre. Réduire les consommations de fluides cryogéniques comme l'Hélium liquide, qui indirectement est un produit pétrolier, par de nouvelles techniques d'isolation et de recyclage du fluide cryogénique. Commuter des gradients de champ très élevés (par exemple 100 $\mathrm{mT} / \mathrm{m}$ ) sur des volumes importants en des temps très courts (inférieurs à $200 \mu \mathrm{s}$ par exemple). Eviter les risques d'échauffement local du patient alors que l'emploi de champs élevés pour l'observation humaine entraîne l'usage de radiofréquences de plus en plus élevées (à 3 teslas, il faut travailler à $130 \mathrm{MHz}$ ). Développer des techniques d'acquisition du signal afin d'éviter au mieux les pertes de rapport signal sur bruit (capteurs en réseaux, techniques de sur-échantillonnage). Créer des approches d'exploration à caractère interventionnel de façon à pouvoir agir directement dans le milieu analysé pendant son observation. Ceci ouvre des portes non seulement en médecine et chirurgie mais aussi dans le domaine des procédés.

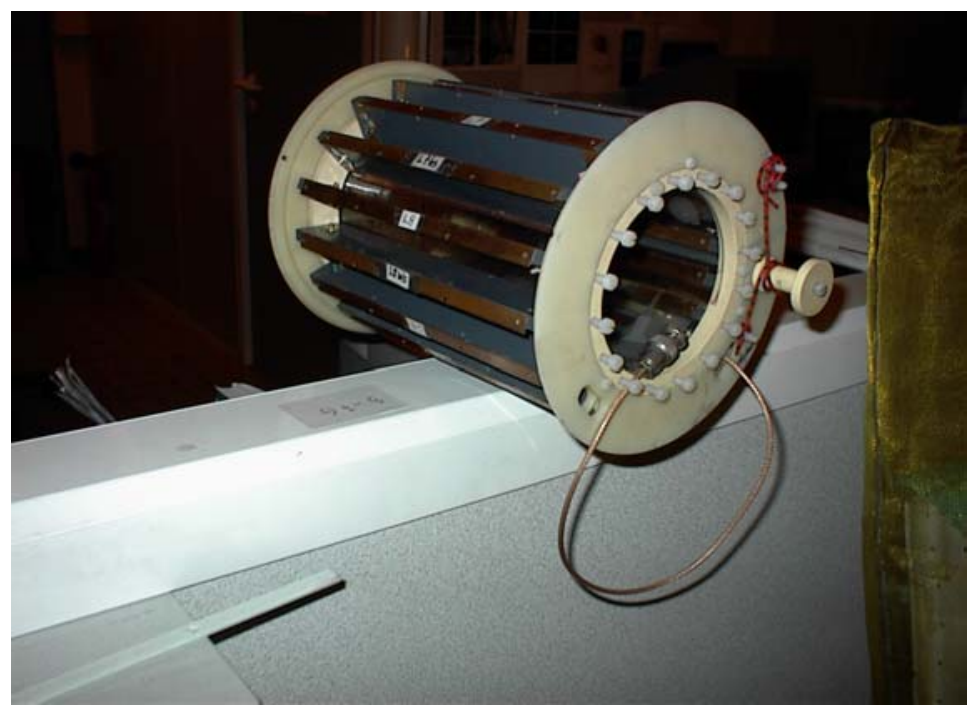

Figure 4 : Résonateur opérant à $85 \mathrm{MHz}$ réalisé par un ensemble de 16 éléments résonnants couplés inductivement entre eux et formant une ligne fermée sur elle même (réalisation Latifa Fakri Bouchet, UMR 5012).

L'enjeu en contrôle non destructif : ce domaine est resté encore assez peu exploré bien que des tentatives aient vu le jour pour des procédés agroalimentaires, pour l'étude d'écoulements mono ou polyphasiques, ou encore pour le test de certains matériaux polymériques qui se prêtent bien à l'expérience. En effet il n'est pas commode de travailler avec des échantillons solides qui donnent des raies de résonance très larges, ce qui nuit à la possibilité de coder aisément l'espace. En outre, les propriétés métalliques ou magnétiques de nombre d'échantillons n'autorisent pas l'emploi de la RMN qui reste essentiellement cantonnée dans l'observation de milieux diamagnétiques et faiblement conducteurs. Loin de tirer un bilan négatif de ces constats, il faut au contraire s'attendre à de plus en plus de tentatives et d'innovation dans un domaine qui commence à être exploré. 

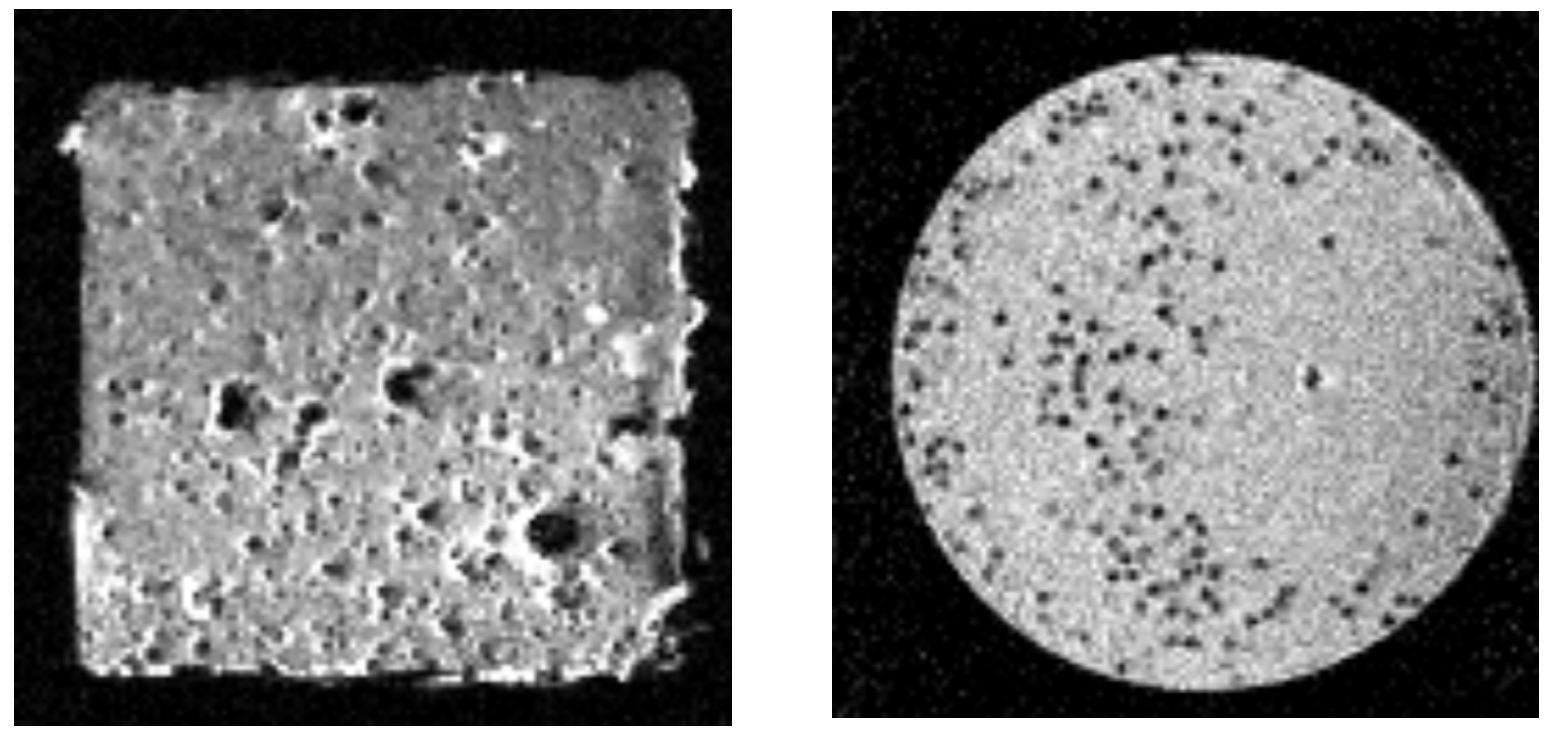

Figure 5 : Exemple d'utilisation de l'imagerie RMN en contrôle non destructif ou en suivi de procédés. A gauche, visualisation d'une matrice polymère servant de réservoir d'huile (produit responsable du niveau de brillance plus ou moins élevé selon sa concentration dans des régions de la coupe qui fait ici $1 \mathrm{~mm}$ d'épaisseur). Dimensions transversales de l'objet : 10×10 mm² (Cliché Rachid Mahdjoub, UMR 5012). A droite, observation d'un phénomène de ségrégation de billes de verres d'un diamètre de $1 \mathrm{~mm}$ lors de l'étude d'un écoulement diphasique de ces billes dans un liquide thixotrope. Epaisseur de coupe $4 \mathrm{~mm}$, Champ de vue $46 \times 46 \mathrm{~mm}^{2}$ (Cliché Rachid Mahdjoub, UMR 5012, et Laurent Jossic, UMR 5520, Laboratoire de Rhéologie, UJF Grenoble).

\section{Références :}

A. BRIGUET. J.L. LEFEVRE "Bases de I'Imagerie par RMN" - L'Onde Electrique $\underline{65}$, $76(1985)$

A. BRIGUET, D. REVEL "La tomographie par Résonance Magnétique Nucléaire" Chapitre 8, Ouvrage sur la Tomographie édité par Pierre Grangeat (Hermès, 2000)

E. M. HAACKE, R.W. BROWN, M.R. THOMPSON, R. VENKATESAN, "Magnetic Resonance Imaging", John Wiley, (1999). 